\title{
Cauchy problem for inhomogeneous parabolic Shilov equations
}

\author{
Dovzhytska I.M.
}

\begin{abstract}
In this paper, we consider the Cauchy problem for parabolic Shilov equations with continuous bounded coefficients. In these equations, the inhomogeneities are continuous exponentially decreasing functions, which have a certain degree of smoothness by the spatial variable. The properties of the fundamental solution of this problem are described without using the kind of equation. The corresponding volume potential, which is a partial solution of the original equation, is investigated. For this Cauchy problem the correct solvability in the class of generalized initial data, which are the Gelfand and Shilov distributions, is determined.

Key words and phrases: parabolic Shilov equation, fundamental solution, Cauchy problem, correct solvability, volume potential.
\end{abstract}

Yuriy Fedkovych Chernivtsi National University, 2 Kotsjubynskyi str., 58012, Chernivtsi, Ukraine

E-mail: i.dovzhytska@chnu.edu.ua

\section{Introduction}

In the famous work by G.Ye. Shilov [1], the definition of parabolicity of systems of equations with partial derivatives, which generalizes the concept of the Petrovsky parabolicity [2], is formulated. It significantly expands the Petrovsky class of parabolic systems of the first-order equations by the time variable with constant coefficients to those systems, in which the order $p$ may no longer coincide with the parabolicity index $h(0<h \leq p)$.

The first studies of parabolic Shilov systems were carried out in [3], where a special method for studying the fundamental solution of such systems, which is based on Phragmén-Lindelöftype theorems, was developed. The classes of unity and correctness of the Cauchy problem using spaces of the Gelfand and Shilov type $S$ were also described. Further research on such systems has been presented in many research papers (see [4-9]). In particular, in $[4,5]$ the properties of the solutions of the Cauchy problem were studied; in [6] the problem of finding the genus $\mu$ of Shilov systems was partially investigated. In $[7,8]$ the alternative methods are suggested for studying the fundamental solution of parabolic Shilov's equations and of the systems that do not require the use of the genus $\mu$. Here also the correct solvability of the Cauchy problem in the classes of Gelfand and Shilov distributions are determined and all classical solutions of Shilov equations in spaces of type $S$ of basic functions are described. The parabolic Shilov abstract differential systems of equations in Banach spaces are investigated in [9]. The main attention in these studies was paid only to the case of constant coefficients. This is primarily due to the fact that Shilov's parabolic systems, in contrast to the systems of Petrovsky, generally speaking, are parabolically unstable to changes in their coefficients, even 
to those found at zero derivative [10].

In [11], the study of parabolic Shilov systems with variable coefficients was started by Ya.I. Zhitomirskiy, where one class of systems parabolically resistant to change of the lower coefficients is proposed, which fully covers the Shilov class. For such systems Ya.I. Zhitomirskiy established the correct solvability of the Cauchy problem in the class of bounded functions. The further study of the Cauchy problem for such and more general systems with nonnegative genus $\mu$ was carried out in [12-14]. Here the fundamental solution of the Cauchy problem is constructed and its main properties within spaces of type $S$ are investigated. Besides, the wide class of the generalized initial data, with which the Cauchy problem for such systems has a unique classical solution, is described.

In these works only homogeneous parabolic equations and systems of equations are studied. In this case, inhomogeneous parabolic Shilov equations and related questions are still waiting for consideration.

This research is devoted to solving the Cauchy problem with generalized initial data, such as the Gelfand and Shilov distributions for parabolic Shilov inhomogeneous equations with time-dependent coefficients. The inhomogeneities in these equations are continuous exponentially decreasing functions, which by the spatial variable have a certain degree of smoothness. Here the correct solvability of this problem is determined, the formula of its solution is found, and the effect of strengthening of the solution convergence when approaching an initial hyperplane is investigated.

The structure of this article is as follows. Section 1 formulates the problem statement and provides the necessary preliminary information. The properties of the fundamental solution of the Cauchy problem for parabolic Shilov equations are investigated in Section 2. Here, developing the method of V.A. Litovchenko [7,8], within spaces of $S$ type the estimates of derivatives of this solution are found, which differ from the existing ones in the fact that they definitely do not contain any kind of equation. Section 3 is devoted to the study of the volume potential of the Cauchy problem. It clarifies the smoothness conditions and the behavior at the density infinity, which provide the required smoothness with respect to the temporal and spatial variables of the corresponding potential of the problem. In addition, its behavior when approaching the initial hyperplane is studied. Sufficient conditions for the correct solvability of the inhomogeneous Cauchy problem are clarified in Section 4. Finally, Section 5 contains the conclusions.

\section{Useful information. Formulation of the problem}

Let us provide the following notations: $\mathbb{R}^{n}$ is a real space of dimension $n \geq 1 ; \mathbb{R}:=\mathbb{R}^{1}$; $\mathbb{Z}_{+}^{n}$ is the set of all $n$-dimensional multiindices; $\mathbb{Z}_{+}:=\mathbb{Z}_{+}^{1} ; i$ is the imaginary unit; $(\cdot, \cdot)$ is the scalar product in $\mathbb{R}^{n} ;\|x\|:=(x, x)^{1 / 2}$ for $x \in \mathbb{R}^{n} ;|x+i y|:=\left(x^{2}+y^{2}\right)^{1 / 2}$, if $\{x, y\} \subset \mathbb{R}$; $z^{l}:=z_{1}^{l_{1}} \ldots z_{n}^{l_{n}}$, if $z:=\left(z_{1} ; \ldots ; z_{n}\right) \in \mathbb{R}^{n}, l:=\left(l_{1} ; \ldots ; l_{n}\right) \in \mathbb{Z}_{+}^{n} ; S$ is the Schwartz space of infinitely differentiable rapidly decreasing functions defined on $\mathbb{R}^{n}$, and $S^{\prime}$ is its topologically dual space [15].

The class of all functions continuously differentiable on $\mathbb{R}^{n}$ up to the order $r$ inclusively is denoted by $\mathbb{C}^{r}\left(\mathbb{R}^{n}\right)$. And let $\mathbb{C}_{l}^{r}\left(\mathbb{R}^{n}\right)$ be the sum of all elements $\varphi$ from $\mathbb{C}^{r}\left(\mathbb{R}^{n}\right)$, so that

$$
\forall k \in \mathbb{Z}_{+}^{n}, \quad|k| \leq r, \quad \exists c_{k}>0 \quad \forall x \in \mathbb{R}^{n}: \quad\left|\partial_{x}^{k} \varphi(x)\right| \leq c_{k}(1+\|x\|)^{-l-|k|} .
$$


Let us put for $\alpha>0$ and $\beta>0$ :

$$
\begin{aligned}
& S_{\alpha}=\left\{\varphi \in \mathbb{C}^{\infty}\left(\mathbb{R}^{n}\right)\left|\exists A>0 \forall k \in \mathbb{Z}_{+}^{n} \exists c_{k}>0 \forall q \in \mathbb{Z}_{+}^{n} \forall x \in \mathbb{R}^{n}:\right| x^{q} \partial_{x}^{k} \varphi(x) \mid \leq c_{k} A^{|q|} q^{\alpha q}\right\} ; \\
& S^{\beta}=\left\{\varphi \in \mathbb{C}^{\infty}\left(\mathbb{R}^{n}\right)\left|\exists B>0 \forall q \in \mathbb{Z}_{+}^{n} \exists c_{q}>0 \forall k \in \mathbb{Z}_{+}^{n} \forall x \in \mathbb{R}^{n}:\right| x^{q} \partial_{x}^{k} \varphi(x) \mid \leq c_{q} B^{|k|} k^{\beta k}\right\} .
\end{aligned}
$$

With the corresponding topologies, the sets $S_{\alpha}$ and $S^{\beta}$ are countably normalized complete perfect spaces, which together with $S_{\alpha}^{\beta}:=S_{\alpha} \cap S^{\beta}$ are referred to as the Gelfand and Shilov type $S$ spaces $[16,17]$.

The $S_{\alpha}^{\beta}$ space is nontrivial at $\alpha+\beta \geq 1$ and contains only functions $\varphi \in \mathbb{C}^{\infty}\left(\mathbb{R}^{n}\right)$ that satisfy the inequality

$$
\left|\partial_{x}^{k} \varphi(x)\right| \leq c B^{|k|} k^{\beta k} e^{-\delta\|x\|^{1 / \alpha}}, \quad k \in \mathbb{Z}_{+}^{n}, \quad x \in \mathbb{R}^{n},
$$

with positive constants $c, B$ and $\delta$, dependent only on the function $\varphi$ [16]. In spaces of $S$ type there are determined and continuous operations of addition, multiplication and convolution, as well as the operator $F$ of Fourier transform, and the following topological equations are satisfied: $F\left[S_{\alpha}\right]=S^{\alpha}, F\left[S^{\beta}\right]=S_{\beta}, F\left[S_{\alpha}^{\beta}\right]=S_{\beta}^{\alpha}$.

Let us consider a differential equation with partial derivatives of order $p>1$

$$
\partial_{t} u(t ; x)=P\left(t ; \partial_{x}\right) u(t ; x)+f(t ; x), \quad(t ; x) \in \Pi_{(0 ;+\infty)}:=(0 ;+\infty) \times \mathbb{R}^{n} .
$$

We assume that the differential expression $P\left(t ; i \partial_{x}\right):=\sum_{|k| \leq p} a_{k}(t) i|k| \partial_{x}^{k}$ on the set $\Pi_{[0 ;+\infty)}$ is uniformly parabolic by Shilov with the parabolicity index $h$, i.e. such that

$$
\exists \delta_{1}>0 \quad \exists \delta_{2} \geq 0 \quad \forall(t ; \xi) \subset \Pi_{[0 ;+\infty)}: \quad \operatorname{Re} \sum_{|k| \leq p} a_{k}(t) \xi^{k} \leq-\delta_{1}\|\xi\|^{h}+\delta_{2},
$$

while the coefficients $a_{k}(\cdot)$ are continuous complex-valued functions on $[0 ;+\infty)$.

By $\Phi^{\prime}$ we denote a topologically dual space to the space $\Phi \in\left\{S^{1 / h} ; S_{\beta}^{1 / h}, \beta \geq(p-1) / h\right\}$. Let us set the initial condition for equation (3)

$$
u(t ;) \underset{t \rightarrow+0}{\longrightarrow} g, \quad g \in \Phi^{\prime} .
$$

Definition. The solution of the Cauchy problem (3), (4) on the set $\Pi_{(0 ; T]}$ is the function $u$, which satisfies equation (3) on $\Pi_{(0 ; T]}$ in the usual sense, and satisfies the initial condition (4) in the sense of convergence in the space $\Phi^{\prime}$.

The fundamental solution of the Cauchy problem for the equation (3) is a function

$$
G(t, \tau ; \cdot)=F^{-1}\left[\theta_{\tau}^{t}(\xi)\right](t, \tau ; \cdot), \quad 0 \leq \tau<t<+\infty,
$$

where

$$
\theta_{\tau}^{t}(\xi)=\exp \left\{\int_{\tau}^{t} P(\varsigma ; \xi) d \varsigma\right\}, \quad P(t ; \xi)=\sum_{|k| \leq p} a_{k}(t) \xi^{k} .
$$

In [7], with the help of Faa de Bruno's formula for differentiation of the compound function, the properties of $\theta_{\tau}^{t}(\cdot)$ are investigated, in particular, it is determined that $\theta_{\tau}^{t}(\cdot)$ belongs to the space $S_{1 / h}^{(p-1) / h}$ for each fixed $t>\tau$ and the following estimates are obtained

$$
\left|\partial_{\tilde{\xi}}^{k} \theta_{\tau}^{t}(\xi)\right| \leq c e^{\delta(t-\tau)} A^{|k|} k^{(p-1) h / k}(t-\tau)^{n+\gamma(k)} e^{-\delta_{0}(t-\tau)\|\xi\|^{h}},
$$

where $k \in \mathbb{Z}_{+}^{n}, \xi \in \mathbb{R}^{n}, 0 \leq \tau<t<+\infty$, with positive constants $c, \delta, \delta_{0}$, and $A$. Here $\gamma(k)=(1-p)|k| / h$, if $0<t-\tau<1$ and $\gamma(k)=(1+h)|k| / h$ at $1 \leq t-\tau$. 
The following statement is correct (see [7]). Let $f \equiv 0$, and $g$ be a real functional from the space $\Phi^{\prime}$. Then the corresponding Cauchy problem (3), (4) on the set $\Pi_{(0 ;+\infty)}$ is correctly solvable; its solution $u(t ; x)$ is differentiable with respect to the variable $t$ and infinitely differentiable with respect to the variable $x$, while the next equality is satisfied

$$
u(t ; x)=\langle g(\xi), G(t, 0 ; x-\xi)\rangle, \quad(t ; x) \in \Pi_{(0 ;+\infty)},
$$

where the angle brackets $\langle\cdot, \cdot\rangle$ indicate the act of a generalized function on a test one.

In the sequel, we will denote the solution of the Cauchy problem (3), (4) for $f \equiv 0$ by $u_{0}$.

Our task is to find out the conditions for the function $f$, under which the corresponding Cauchy problem (3), (4) will have a unique classical solution.

Taking into consideration the linearity of the equation (3) and having the information about the correct solvability of the Cauchy problem (3), (4) for $f \equiv 0$, it is expedient to search for the solution of this problem for the inhomogeneous equation (3) in the form of the sum $u=u_{0}+u_{1}$, where $u_{1}$ is the solution of equation (3), which satisfies the initial condition (4) for $g=0$, i.e.

$$
u_{1}(t ;) \underset{t \rightarrow+0}{\longrightarrow} 0 .
$$

If equation (3) is parabolic by Petrovsky, then the solution of the Cauchy problem (3), (7) is determined by the following formula $[18,19]$ :

$$
u_{1}(t ; x)=\int_{0}^{t} d \tau \int_{\mathbb{R}^{n}} G(t, \tau ; x-\xi) f(\tau ; \xi) d \xi, \quad(t ; x) \in \Pi_{(0 ;+\infty)} .
$$

Thus, our task was reduced to the study of the properties of the corresponding volume potential (8) for the parabolic Shilov equation (3). To do that, first we find out the properties of the fundamental solution $G(t, \tau ; \cdot)$.

\section{Fundamental solution of the Cauchy problem}

Taking into account the representation (5) of the function $G(t, \tau ; \cdot)$, as well as belonging of $\theta_{\tau}^{t}(\cdot)$ to $S_{1 / h}^{(p-1) / h}$ and properties of spaces of the $S$ type, we obtain that for fixed $t$ and $\tau$ the fundamental solution $G(t, \tau ; \cdot)$ is an element of the space $S_{1 / h}^{(p-1) / h}$. Then for the derivatives $\partial_{x}^{k} G(t, \tau ; \cdot)$ the corresponding estimates (2) must be satisfied. We find these estimates, emphasizing the dependence on the variables $t$ and $\tau$.

As far as the representation is performed

$$
(i x)^{q} \partial_{x}^{k} G(t, \tau ; x)=(2 \pi)^{-n} \int_{\mathbb{R}^{n}} e^{-i(x, \xi)} \partial_{\xi}^{q}\left((i \xi)^{k} \theta_{\tau}^{t}(\xi)\right) d \xi, \quad x \in \mathbb{R}^{n}, \quad 0 \leq \tau<t, \quad\{q, k\} \subset \mathbb{Z}_{+}^{n},
$$

then, according to the Leibniz formula of the product of functions differentiation, we obtain

$$
\left|x^{q} \partial_{x}^{k} G(t, \tau ; x)\right| \leq(2 \pi)^{-n} \sum_{l=0}^{q} C_{q}^{l} \int_{\mathbb{R}^{n}}\left|\partial_{\xi}^{l} \xi^{k}\right|\left|\partial_{\xi}^{q-l} \theta_{\tau}^{t}(\xi)\right| d \xi, \quad x \in \mathbb{R}^{n}, \quad 0 \leq \tau<t, \quad\{q, k\} \subset \mathbb{Z}_{+}^{n},
$$

here $C_{q}^{l}$ is a binomial coefficient.

Hence, using the estimates (6) and taking into account the equations

$$
\partial_{\xi}^{l} \xi^{k}=\frac{k !}{(k-l) !} \prod_{j=1}^{n}\left\{\begin{array}{ll}
\xi_{j}^{k_{j}-l_{j}}, & l_{j} \leq k_{j}, \\
0, & k_{j}<l_{j},
\end{array} \quad \text { and } \quad \sup _{\rho \geq 0}\left\{\rho^{\alpha} e^{-\delta \rho}\right\}=\left(\frac{\alpha}{e \delta}\right)^{\alpha}, \quad \alpha>0, \quad \delta>0,\right.
$$


we obtain for $x \in \mathbb{R}^{n}, 0 \leq \tau<t$, and $\{q, k\} \subset \mathbb{Z}_{+}^{n}$,

$$
\begin{aligned}
\left|x^{q} \partial_{x}^{k} G(t, \tau ; x)\right| \leq c e^{\delta(t-\tau)} \sum_{l=0}^{q} C_{q}^{l} C_{k}^{l} l ! A^{|q-l|}(q-l)^{(p-1)(q-l) / h}(t-\tau)^{n+\gamma(q-l)} & \\
& \times \int_{\mathbb{R}^{n}}|\xi|^{k-l} e^{-\delta_{0}(t-\tau)\|\xi\|^{h}} d \xi \\
\leq & c e^{\delta(t-\tau)} \sum_{l=0}^{q} C_{q}^{l} C_{k}^{l} l ! A^{|q-l|}(q-l)^{(p-1)(q-l) / h}(t-\tau)^{n+\gamma(q-l)-(n+|k-l|) / h} \\
& \times \prod_{j=1}^{n} \sup _{j}\left\{\rho_{j}^{\left(k_{j}-l_{j}\right) / h} e^{-\left(\delta_{0} \rho_{j}\right) /(2 n)}\right\} \int_{\mathbb{R}^{n}} e^{-\left(\delta_{0} / 2\right)\|y\|^{h}} d y \\
\leq & e^{\delta(t-\tau)}(t-\tau)^{n+\gamma(q)-(n+|k|) / h} c_{1} A_{1}^{|q|} B_{1}^{|k|} q^{(p-1) q / h} k^{k / h},
\end{aligned}
$$

here positive values $c_{1}, A_{1}, B_{1}$ and $\delta$ do not depend on $t, \tau, x, q$ and $k$.

From the last ratio, we come to the following estimate

$$
\left|\partial_{x}^{k} G(t, \tau ; x)\right| \leq e^{\delta(t-\tau)}(t-\tau)^{n-(n+|k|) / h} c_{1} B_{1}^{|k|} k^{k / h} \prod_{j=1}^{n} \inf _{q_{j} \geq 0}\left\{\left(A_{j}\left|x_{j}\right|^{-1} q_{j}^{(p-1) / h}\right)^{q_{j}}(t-\tau)^{\gamma\left(q_{j}\right)}\right\} .
$$

Thus, the following statement is true.

Lemma 1. There are positive constants $c, B, \delta$ and $\delta_{0}$ such that for all $x \in \mathbb{R}^{n}, k \in \mathbb{Z}_{+}^{n}$ and $0 \leq \tau<t$ the following estimates are performed

$$
\left|\partial_{x}^{k} G(t, \tau ; x)\right| \leq e^{\delta(t-\tau)}(t-\tau)^{n-(n+|k|) / h_{c}} B^{|k|} k^{\frac{k}{h}} \exp \left\{-\delta_{0}\left(\frac{\|x\|^{h}}{(t-\tau)^{\gamma_{0}}}\right)^{1 /(p-1)}\right\},
$$

where $\gamma_{0}=1-p$, if $0<t-\tau<1$ and $\gamma_{0}=1+h$ at $1 \leq t-\tau$.

Note here that the estimates (9) of the fundamental solution $G$ in comparison with the estimates established in $[3,11]$ do not contain the genus $\mu$ of the equation (3), therefore this allows us to avoid the problems associated with finding this characteristic.

The " $\delta$-similarity" of the function $G(t, \tau ; \cdot)$ is characterized by the following auxiliary statement.

Lemma 2. Let $\varphi(\cdot) \in \mathbb{C}_{l}^{r}\left(\mathbb{R}^{n}\right)$, then for $l>n$ and $r>n$ each of the following boundary relations

$$
(G * \varphi)(t, \tau ; \cdot) \underset{t \rightarrow \tau+0}{\stackrel{x \in \mathbb{K}}{\rightrightarrows}} \varphi(\cdot) ; \quad(G * \varphi)(t, \tau ; \cdot) \underset{t \rightarrow \tau+0}{\stackrel{x \in \mathbb{K}}{\rightrightarrows}} \varphi(\cdot)
$$

is satisfied; here we mean the uniform convergence on each compact set $\mathbb{K} \subset \mathbb{R}^{n}$.

Proof. Since $\varphi \in \mathbb{C}_{l}^{r}\left(\mathbb{R}^{n}\right)$, then for $l>n$ there is a Fourier transform $F[\varphi]$ and the estimate is performed

Then the correct image is

$$
|F[\varphi](\xi)| \leq \frac{c_{0}}{(1+\|\xi\|)^{r}}, \quad \xi \in \mathbb{R}^{n}
$$

$$
(G * \varphi)(t, \tau ; x) \equiv \int_{\mathbb{R}^{n}} G(t, \tau ; x-\xi) \varphi(\xi) d \xi=(2 \pi)^{-n} \int_{\mathbb{R}^{n}} \theta_{\tau}^{t}(y) F[\varphi](y) e^{-i(x, y)} d y .
$$

The uniform convergence

$$
\theta_{\tau}^{t}(x) F[\varphi](x) \underset{t \rightarrow \tau+0}{\stackrel{x \in \mathbb{K}}{\rightrightarrows}} F[\varphi](x)
$$


and the fact that

$$
\exists c>0 \quad \forall t-\tau<1 \quad \forall x \in \mathbb{R}^{n}: \quad\left|\theta_{\tau}^{t}(x) F[\varphi](x)\right| \leq \frac{c e^{\delta_{2}}}{(1+\|x\|)^{r}},
$$

ensures the correctness of the equality

$$
\lim _{t \rightarrow \tau+0} \int_{\mathbb{R}^{n}} \theta_{\tau}^{t}(y) F[\varphi](y) e^{-i(x, y)} d y=\int_{\mathbb{R}^{n}} \lim _{t \rightarrow \tau+0} \theta_{\tau}^{t}(y) F[\varphi](y) e^{-i(x, y)} d y=(2 \pi)^{n} F^{-1}[F[\varphi]](x) .
$$

Hence, taking into account the reversibility of the operator $F$ on the elements of the class $\mathbb{C}_{l}^{r}\left(\mathbb{R}^{n}\right)$ for $l>n$ and $r>n$, we arrive at the first boundary relation (10).

The second boundary relation (10) can be proved similarly.

We are going to study the properties of the volume potential (8).

\section{Volume potential of the Cauchy problem}

We start with formulating the conditions for the density $f$, under which we investigate the potential (8).

We say that for a function $f(t ; x)$ on the set $\Pi_{[0 ;+\infty)}$ the condition $(A)$ is satisfied, if $f$ is continuous on $\Pi_{[0 ;+\infty)}$ and $f(t ; \cdot) \in \mathbb{C}_{l}^{r}\left(\mathbb{R}^{n}\right), t \geq 0$, and value $c_{k}(\cdot)$ from the corresponding estimate (1) is limited on each compact $\mathbb{K} \subset[0 ;+\infty)$, i.e. $\sup _{t \in \mathbb{K}} c_{k}(t)<\infty$.

Theorem 1. Let $h>n /(n+1)$ and for a function $f$ the condition $(A)$ is satisfied for $l>n$, then the corresponding potential $u_{1}(t ; \cdot)$ for each fixed $t>0$ is a differentiable function on the set $\mathbb{R}^{n}$ up to the order $r$ inclusive, for derivatives of which the formula below is correct

$$
\partial_{x}^{k} u_{1}(t ; x)=\int_{0}^{t} d \tau \int_{\mathbb{R}^{n}} G(t, \tau ; \xi) \partial_{x}^{k} f(\tau ; x-\xi) d \xi, \quad(t ; x) \in \Pi_{(0 ;+\infty)} .
$$

Proof. Let us use the representation

$$
u_{1}(t ; x)=\int_{0}^{t} d \tau \int_{\mathbb{R}^{n}} G(t, \tau ; \xi) f(\tau ; x-\xi) d \xi, \quad(t ; x) \in \Pi_{(0 ;+\infty)},
$$

from which, by formal differentiation under the integral sign, we arrive at the formula (11).

Then, to substantiate the equality (11), it is enough to prove the uniform convergence with respect to the variable $x$ on the set $\mathbb{R}^{n}$ of the integral

$$
\Im_{k}(t ; x)=\int_{0}^{t} d \tau \int_{\mathbb{R}^{n}}|G(t, \tau ; \xi)|\left|\partial_{x}^{k} f(\tau ; x-\xi)\right| d \xi, \quad|k| \leq r .
$$

However, this convergence becomes obvious if we consider condition (A) and the estimate (9), according to which for all $(t ; x) \in \Pi_{[0 ;+\infty)}$ and $|k| \leq r$ the inequality below is satisfied

$$
\mathfrak{I}_{k}(t ; x) \leq c_{k} e^{\delta t} \int_{0}^{t}(t-\tau)^{n-n / h} d \tau \int_{\mathbb{R}^{n}} \frac{d \xi}{(1+\|x-\xi\|)^{l+|k|}} \equiv \frac{c_{k} e^{\delta t} h t^{n-n / h+1}}{h(n+1)-n} \int_{\mathbb{R}^{n}} \frac{d y}{(1+\|y\|)^{l+|k|}},
$$

under the condition that $h>n /(n+1)$ and $l>n$.

Corollary 1. If for the equation (3) the parabolicity index $h$ is greater than $n /(n+1)$, and the inhomogeneity $f$ satisfies the condition $(A)$ for $l>n$ and $r \geq p$, then

$$
P\left(t ; i \partial_{x}\right) u_{1}(t ; x)=\sum_{|k| \leq p} a_{k}(t) i^{|k|} \int_{0}^{t} d \tau \int_{\mathbb{R}^{n}} G(t, \tau ; \xi) \partial_{x}^{k} f(\tau ; x-\xi) d \xi, \quad(t ; x) \in \Pi_{(0 ;+\infty)} .
$$


The differentiability of the function $u_{1}$ by the variable $t$ characterizes the following statement.

Theorem 2. Let $h>\frac{n}{n+1}$ and for the function $f$ the condition $(A)$ is satisfied for $l>n$ and $r>\max \{n, p-1\}$, then the corresponding potential $u_{1}$ on the set $\Pi_{(0 ;+\infty)}$ is a differentiable function of the variable $t>0$, and the equality below is satisfied

$$
\partial_{t} u_{1}(t ; x)=f(t ; x)+\sum_{|k| \leq p} a_{k}(t) i^{|k|} \int_{0}^{t} d \tau \int_{\mathbb{R}^{n}} G(t, \tau ; \xi) \partial_{x}^{k} f(\tau ; x-\xi) d \xi, \quad(t ; x) \in \Pi_{(0 ;+\infty)} .
$$

Proof. We arbitrarily fix $t>0$ and consider the auxiliary function

$$
u_{1}^{\varepsilon}(t ; x)=\int_{0}^{t-\varepsilon} d \tau \int_{\mathbb{R}^{n}} G(t, \tau ; x-\xi) f(\tau ; \xi) d \xi, \quad x \in \mathbb{R}^{n}, \quad 0<\varepsilon<t / 2 .
$$

It is obvious that

$$
\partial_{t} u_{1}^{\varepsilon}(t ; x)=\int_{\mathbb{R}^{n}} G(t, t-\varepsilon ; x-\xi) f(t-\varepsilon ; \xi) d \xi+\int_{0}^{t-\varepsilon} d \tau \int_{\mathbb{R}^{n}} \partial_{t} G(t, \tau ; x-\xi) f(\tau ; \xi) d \xi .
$$

We now find the limit $\lim _{\varepsilon \rightarrow+0} \partial_{t} u_{1}^{\varepsilon}(t ; x)$. Taking into account the properties of the function $f$, directly from the statement of Lemma 2 we obtain that

$$
\int_{\mathbb{R}^{n}} G(t, t-\varepsilon ; x-\xi) f(t-\varepsilon ; \xi) d \xi \underset{\varepsilon \rightarrow+0}{\rightarrow} f(t ; x) .
$$

Further, since $G$ is the solution of the equation (3), then

$$
\int_{0}^{t-\varepsilon} d \tau \int_{\mathbb{R}^{n}} \partial_{t} G(t, \tau ; x-\xi) f(\tau ; \xi) d \xi=\sum_{|k| \leq p} a_{k}(t) i^{|k|} \int_{0}^{t-\varepsilon} d \tau \int_{\mathbb{R}^{n}} \partial_{x-\xi}^{k} G(t, \tau ; x-\xi) f(\tau ; \xi) d \xi .
$$

After replacing the integration variable in the last integral by the rule $y=x-\xi$, and then, integrating by parts $k$ times, we arrive at the following equality

$$
\int_{0}^{t-\varepsilon} d \tau \int_{\mathbb{R}^{n}} \partial_{x-\xi}^{k} G(t, \tau ; x-\xi) f(\tau ; \xi) d \xi=\int_{0}^{t-\varepsilon} d \tau \int_{\mathbb{R}^{n}} G(t, \tau ; y) \partial_{x-y}^{k} f(\tau ; x-y) d y .
$$

Using the estimate (9) for the fundamental solution $G$ and considering the fulfillment of condition $(A)$ for the function $f$, we find:

$$
\begin{aligned}
& \left|\int_{t-\varepsilon}^{t} d \tau \int_{\mathbb{R}^{n}} G(t, \tau ; y) \partial_{x-y}^{k} f(\tau ; x-y) d y\right| \\
& \quad \leq c_{k} e^{\delta t} \int_{t-\varepsilon}^{t}(t-\tau)^{n-n / h} d \tau \int_{\mathbb{R}^{n}} \frac{d y}{(1+\|y\|)^{l+|k|}}=\hat{c}_{k} e^{\delta t} \varepsilon^{n+1-n / h} .
\end{aligned}
$$

The obtained estimate ensures that the limit relation is correct for $h>n /(n+1)$

$$
\lim _{\varepsilon \rightarrow+0} \int_{0}^{t-\varepsilon} d \tau \int_{\mathbb{R}^{n}} \partial_{x-\xi}^{k} G(t, \tau ; x-\xi) f(\tau ; \xi) d \xi=\int_{0}^{t} d \tau \int_{\mathbb{R}^{n}} G(t, \tau ; y) \partial_{x-y}^{k} f(\tau ; x-y) d y,
$$

and, consequently, the fulfillment of the equality (12).

We further clarify the question of the existence of a limit value of the potential $u_{1}$ on the initial hyperplane $t=0$. 
Assuming that $h>n /(n+1)$ and for the function $f$ the condition $(A)$ is fulfilled for $l>n$, then according to the estimate (9), for all $(t ; x) \in \Pi_{(0 ;+\infty)}$ we have

$$
\begin{aligned}
\left|u_{1}(t ; x)\right| & \leq \int_{0}^{t} d \tau \int_{\mathbb{R}^{n}}|G(t, \tau ; x-\xi)||f(\tau ; \xi)| d \xi \\
& \leq c e^{\delta t} \int_{0}^{t}(t-\tau)^{n-n / h} d \tau \int_{\mathbb{R}^{n}} \frac{d \xi}{(1+\|\xi\|)^{l}}=\hat{c} e^{\delta t} t^{n+1-n / h} .
\end{aligned}
$$

From this we obtain that the limit relation (7) is satisfied, and in this case, $u_{1}$ tends to zero that occurs uniformly with respect to the variable $x$ on $\mathbb{R}^{n}$.

Therefore, the following statement is correct.

Theorem 3. Assuming that $h>n /(n+1)$ and for the function $f$ the condition $(A)$ is fulfilled for $l>n$, then for the corresponding potential $u_{1}(t ; \cdot)$ the ratio below is correct

$$
u_{1}(t ; x) \underset{t \rightarrow+0}{\stackrel{x \in \mathbb{R}^{n}}{\rightrightarrows}} 0 .
$$

In the next section, the Cauchy problem for the inhomogeneous equation (3) is considered.

\section{Cauchy problem}

The previously obtained information on the volume potential $u_{1}$ allows us to draw certain conclusions about the correct solvability of the inhomogeneous Cauchy problem for parabolic Shilov equations.

Theorem 4. Let $g$ be a real-valued functional from the space $\Phi^{\prime}$, while $h>n /(n+1)$, and for the function $f$ the condition $(A)$ is fulfilled for $l>n$ and $r>\max \{n, p-1\}$, then the corresponding Cauchy problem (3), (4) on the set $\Pi_{(0 ;+\infty)}$ is correctly solvable. Its solution $u$ is once differentiable by the variable $t$ and $r$ times - by the variable $x$, and is represented by the formula

$$
u(t ; x)=\langle g(\xi), G(t, 0 ; x-\xi)\rangle+\int_{0}^{t} d \tau \int_{\mathbb{R}^{n}} G(t, \tau ; x-\xi) f(\tau ; \xi) d \xi, \quad(t ; x) \in \Pi_{(0 ;+\infty)} .
$$

Proof. We write the equation (13) in a compact form: $u=u_{0}+u_{1}$. The smoothness of the function $u$ indicated in the formulation of Theorem 4 follows from the smoothness of the function $u_{0}$ [7] and the statements of Theorems 1, 2.

Directly from Corollary 1, the equality (12), and the statements of Theorems 2, 3, we obtain that $u_{1}$ is the solution of the Cauchy problem (3), (7). Then $u$ is the classical solution of the Cauchy problem (3), (4) on the set $\Pi_{(0 ;+\infty)}$.

Let us substantiate the unity of the solution of this problem. We assume that there are two solutions of the Cauchy problem (3), (4): $\hat{u}$ and $\breve{u}$. Then their difference $u=\hat{u}-\check{u}$ will be the solution of a homogeneous problem:

$$
\partial_{t} u(t ; x)=P\left(t ; \partial_{x}\right) u(t ; x) ; \quad u(t ; \cdot)_{t=0}=0 .
$$

However, this problem has only a zero solution [7]: $u=0$. Therefore, $\hat{u}=\breve{u}$, and the Cauchy problem (3), (4) on the set $\Pi_{(0 ;+\infty)}$ has a unique solution (13). This solution continuously depends on the initial data, because this is the solution $u_{0}$ of the Cauchy problem (3), (4) for $f=0$ [7]. 
As it has already been mentioned, the initial condition (4) is regarded in the sense of weak convergence in the space $\Phi^{\prime}$, because the initial function $g$ is a functional from $\Phi^{\prime}$. However, if this functional has "good" properties, then the effect of the convergence increase in the condition (4) can be observed. In particular, if $g$ is a regular generalized function generated by the ordinary function $g(\cdot)$ from the class $\mathbb{C}_{l}^{r}\left(\mathbb{R}^{n}\right)$, then

$$
u_{0}(t ; x)=\int_{\mathbb{R}^{n}} G(t, 0 ; x-\xi) g(\xi) d \xi, \quad(t ; x) \in \Pi_{(0 ;+\infty)},
$$

and provided that $l>n$ and $r>n$, the initial condition (4) can already be considered as a uniform convergence with respect to the spatial variable $x$ on each compact set $\mathbb{K} \subset \mathbb{R}^{n}$ :

$$
u(t ; x) \underset{t \rightarrow+0}{\stackrel{x \in \mathbb{K}}{\rightrightarrows}} g(x) .
$$

This fact becomes obvious if we consider the statements of Lemma 2, Theorem 3, and the fact that $u=u_{0}+u_{1}$.

\section{Conclusions}

Sufficient conditions for the inhomogeneity of parabolic Shilov equations with variable coefficients are found, according to which the Cauchy problem for such equations in the class of generalized initial data of the Gelfand and Shilov distributions has a unique classical solution that continuously depends on the initial data. The obtained results, in addition to filling to some extent the corresponding gaps in the theory of the Cauchy problem for parabolic Shilov equations, allow to develop this theory for equations with quasilinear structure in the classical way. In addition, they will find their application in solving inhomogeneous parabolic equations of the Shilov type with space-dependent coefficients as well as in the study of the properties of their solutions, etc.

Further development of the problem is represented by defining the conditions of the inhomogeneity of such equations, which provide the appropriate solution for the presence of certain properties, in particular, its belonging to spaces of the $S$ type or its stabilization at infinity.

\section{References}

[1] Shilov G.E. On conditions of correctness of Cauchy's problem for systems of partial differential equations with constant coefficients. Uspekhi Mat. Nauk 1955, 10 (4), 89-100. (in Russian)

[2] Petrovskii I.G. On the Cauchy problem for systems of equations with partial derivatives in a domain of nonanalytic functions. Bull. Mosk. Gos. Univ. Ser. Mat. Mekh. 1938, 1 (7), 1-72. (in Russian)

[3] Gel'fand I., Shilov G. Generalized functions. Vol. 3. Theory of differential equations. Academic Press, New York, 1967.

[4] Eidelman S.D., Ivasishen S.D., Porper F.O. The Liouville theorems for systems parabolic by Shilov. Izv. Vyssh. Uchebn. Zaved. Mat. 1961, 6, 169-179. (in Russian)

[5] Gorodetskii V.V. Localization principle for solutions of the Cauchy problem for parabolic systems in the class of generalized functions of infinite order. Differ. Uravn. 1985, 21 (6), 1077-1079. (in Russian)

[6] Boroc V.M. Reduction of a system of linear equations in partial derivatives with constant coefficients to the canonical canonical form. Dokl. Akad. Nauk SSSR 1957, 115 (1), 13-16. (in Russian) 
[7] Litovchenko V. The Cauchy problem for Shilov parabolic equations. Sib. Math. J. 2004, 45 (4), $669-679$. doi:10.1023/B:SIMJ.0000035831.63036.bb (translation of Sibirsk. Mat. Zh. 2004, 45 (4), 809-821. (in Russian))

[8] Litovchenko V. Cauchy problem for $\{\vec{p} ; \vec{h}\}$-parabolic equations with time-dependent coefficients. Math. Notes 2005, 77 (3-4), 364-379. doi:10.1007/s11006-005-0036-9

[9] Kostić M. Shilov parabolic systems. Bull. Cl. Sci. Math. Nat. Sci. Math. 2012, 37, 19-39.

[10] Hou-Sin U. On the definition of parabolic systems of partial differential equations. Uspekhi Mat. Nauk 1960, 15 (6), 157-161. (in Russian)

[11] Zhitomirskii Ya.I. Cauchy problem for some types of systems of linear equations, which are parabolic by G.E. Shilov, with partial derivatives with continuous coefficients. Izv. Akad. Nauk SSSR Ser. Mat. 1959, 23, 925-932. (in Russian)

[12] Litovchenko V., Dovzhytska I. Cauchy problem for a class of parabolic systems of Shilov type with variable coefficients. Cent. Eur. J. Math. 2012, 10 (3), 1084-1102. doi:10.2478/s11533-012-0025-7

[13] Litovchenko V.A., Unguryan G.M. Conjugate Cauchy problem for parabolic Shilov type systems with nonnegative genus. Differ. Equ. 2018, 54 (3), 335-351. doi:10.1134/S0012266118030060 (translation of Differ. Uravn. 2018, 54 (3), 341-357. (in Russian))

[14] Litovchenko V., Unguryan G. Some properties of Green's functions of Shilov-type parabolic systems. Miskolc Math. Notes 2019, 20 (1), 365-379. doi:10.18514/MMN.2019.2089

[15] Schwartz L. Théorie des distributions. Hermann, Paris, 1966.

[16] Gel'fand I.M., Shilov G.E. Spaces of fundamental and generalized functions. Fizmatlit, Moscow, 1958. (in Russian)

[17] Kashpirovsky A.I. Equality of the spaces $S_{\alpha}^{\beta}$ and $S_{\alpha} \cap S^{\beta}$. Funct. Anal. Appl. 1980, 14 (2), 129-129. doi: 10.1007/BF01086561 (translation of Funkts. Anal. Prilozh. 1980, 14 (2), 60-60. (in Russian))

[18] Eidelman S.D. Parabolic systems. North-Holland, Amsterdam, 1969.

[19] Friedman A. Partial differential equations of parabolic type. Prentice-Hall, Englewood Cliffs, 1964.

Received 26.01.2021

Revised 13.03.2021

Аовжицька I.М. Задача Комі для неоднорідних параболічних за Шиловим рівнянь // Карпатські матем. публ. - 2021. — Т.13, №2. — С. 475-484.

У даній роботі розглядається задача Коші для параболічних за Шиловим рівнянь 3 неперервними обмеженими коефіцієнтами, неоднорідності яких є класичними функціями, що степенево спадають на нескінченності і мають за просторовою змінною певний ступінь гладкості. Описано властивості фундаментального розв'язку цієї задачі без використання роду рівняння та досліджено відповідний об'ємним потенціал, шо $є$ частинним розв'язком вихіАного неоднорідного рівняння. Аля таких рівнянь знайдено класичні розв'язки, граничні значення яких на початковій гіперплощині можуть бути узагальненими функціями типу розподілів Гельфанда і Шилова та обгрунтовано їх єлиність і неперервну залежність від початкових даних.

Ключові слова і фрази: параболічне за Шиловим рівняння, фундаментальний розв'язок, задача Коші, коректна розв' язність, об'ємний потенціал. 